\title{
Deutsche Kardiodiagnostiktage (DKDT) 2017 in Leipzig: Bildgebung im aktuellen Fokus
}

Die 9. Deutschen Kardiodiagnostiktage haben vom 23.-25. Februar wieder in Leipzig stattgefunden. Mit fast 600 Teilnehmern gehören die Deutschen Kardiodiagnostiktage mittlerweile zu den führenden kardiovaskulären Bildgebungssymposien in Europa und werden interdisziplinär unter der Ägide der Arbeitsgruppen für CT und MRT der Deutschen Gesellschaft für Kardiologie - Herz- und Kreislaufforschung e. V. (DGK) und der AG Herz- und Gefäßdiagnostik der Deutschen Röntgengesellschaft e. V. (DRG) organisiert. Ebenso erhält die Veranstaltung die aktive Unterstützung der AG Nuklearkardiologie der Deutschen Gesellschaft für Nuklearmedizin e. V. (DGN). An 3 Tagen wurden Entwicklungen, Trends und aktuelle Studienergebnisse auf dem Gebiet der kardiovaskulären Bildgebung diskutiert.

Traditionell fanden am ersten Tag Workshops zur kardialen Magnetresonanztomografie (MRT) und der Computertomografie (CT) statt, die entsprechend des unterschiedlichen Levels zertifiziert und anerkannt sind. Zwischen den einzelnen Workshops wurden multiple LIVE-Cases gezeigt, welche die Notwendigkeit der additiven Bildgebung wie der Echokardiografie, der kardialen MRT, der CT bei kardiovaskulären Interventionen, wie TAVI oder MitraClip oder bei elektrophysiologischen Eingriffen unterstrichen. Im letzten Jahr standen u. a. LIVECases zur nicht-invasiven Koronardiagnostik im Vergleich zur FFR-Bestimmung mittels Computational Fluid Dynamics (CFD) durch $\mathrm{CT}_{\mathrm{FFR}}$ oder QFR im Vordergrund, zu denen noch Ergebnisse nachgetragen werden konnten. So stimmten die Ergebnisse der LIVE invasiv gemessenen FFR gut mit den Ergebnissen der QFR aus der Angio und der $\mathrm{CT}_{\text {FFR }}$ aus der Koronar-CT bei einer Patientin mit hochgradiger distaler RCA-Stenose überein. Alle Berechnungen zeigten eine FFR oberhalb des Normbereichs, weshalb von ei-

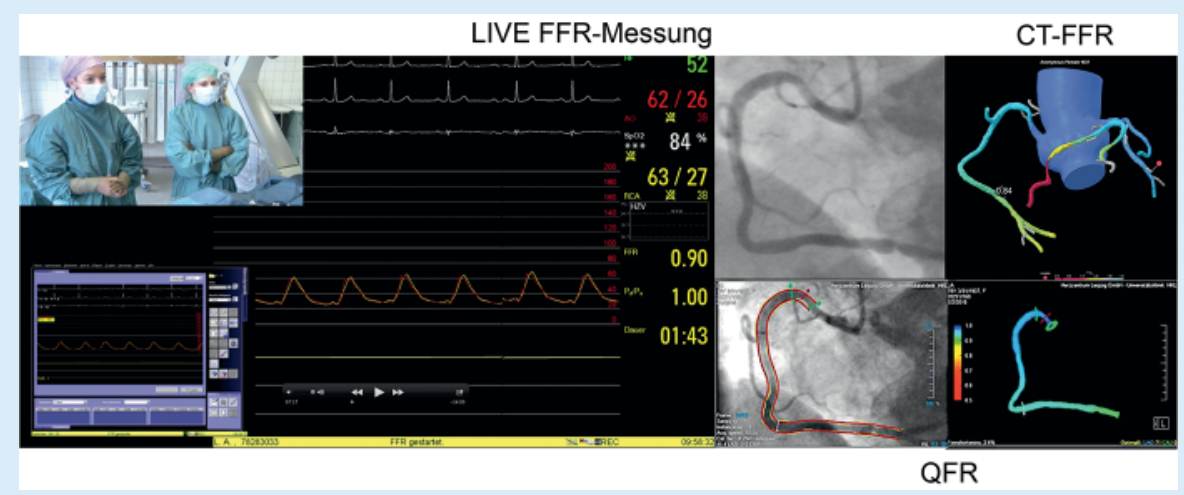

- Abb. 1 „Warten auf den FFR-Abfall“. Nachtrag zum KHK-LIVE-CASE DKDT 2016. Sowohl in der QFR (Fa. Medis) aus den biplanen Angiografiedaten, als auch in der nachträglich bestimmten CTFFR (Fa. HeartFlow) aus CT-Daten konnte korrespondierend zu den invasiv ermittelten Werten keine hämodynamische Relevanz einer visuell hochgradigen distalen RCA-Stenose nachgewiesen werden.

ner interventionellen Therapie abgesehen werden konnte ( $\triangleright$ Abb. 1).

An den beiden Folgetagen standen nach der offiziellen Eröffnung durch die beiden Kongresspräsidenten - Prof. Holger Thiele (Lübeck) und Prof. Matthias Gutberlet (Leipzig) - und die traditionellen Ehrung eines Vertreters aus der Kardiologie oder Radiologie, der sich um die kardiovaskuläre Bildgebung besonders verdient gemacht hat, der Austausch zu aktuellen wissenschaftlichen Ergebnissen und die Umsetzung der Ergebnisse im klinischen Alltag im Vordergrund. In diesem Jahr wurde Prof. Dr. med. Dr. h. c. Maximilian Reiser (München) geehrt ( $\triangleright$ Abb. 2).

In der kardialen MRT stellt die parametrische Bildgebung (Mapping) derzeit das „Hot Topic" schlechthin dar. Hiermit werden für kardiale Gewebe spezifische, absolut quantifizierbare T1- oder T2-Zeiten für jedes Voxel, generiert und somit z. B. Informationen über die Myokardfibrosierung oder Inflammation erhalten. Die klinischen Einsatzmöglichkeiten des Mapping sind vielfältig. In aktuellen

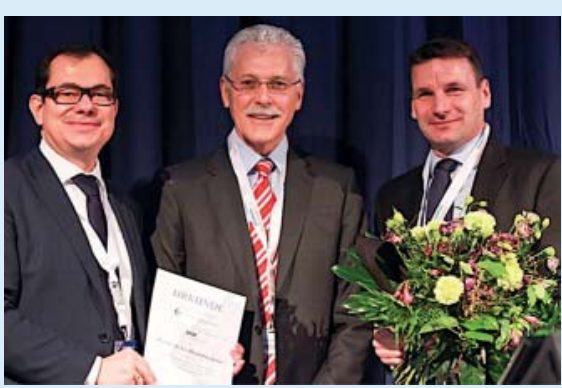

Abb. 2 Prof. Dr. med. Matthias Gutberlet (links) und Prof. Dr. med. Holger Thiele (rechts) überreichen Prof. Dr. med. Dr. h. c. Maximilian Reiser (Mitte) stellvertretend für die Präsidenten der DRG und DGK die Urkunde für seine Verdienste um die nichtinvasive kardiovaskuläre Bildgebung.

Studien konnte gezeigt werden, dass mittels T1- und T2-Mapping zum Beispiel die Diagnosestellung bei der Myokarditis im Vergleich zu den Standard Lake Louise-Kriterien [1], die eine Kombination aus frühem relativem Enhancement, relativem Wassergehalt und delayed enhancement nutzen, verbessert werden kann ( $\triangleright$ Abb. 3) [2]. In einer impulsiv geführten Pro- und Kontra-Debatte wurde ausführlich die Frage nach dem geeig- 
netsten Referenzstandard für die Diagnose einer Myokarditis diskutiert. In vielen Studien wurde alleine eine klinische Einschätzung als Referenzstandard gewählt, während für viele Autoren der Referenzstandard eher die endomyokardiale Biopsie darstellt, auch wenn selbst für die histopathologische Analyse die Kriterien für die Diagnosestellung nicht $100 \%$ validiert sind. An der regen Diskussion beteiligten sich u. a. auch zwei Pioniere der MRT-Myokarditisdiagnostik, Frau Prof. Jeanette Schulz-Menger (Berlin) und Prof. Matthias Friedrich (Montreal/Heidelberg), die sich für eine alleinige klinische Einschätzung, insbesondere bei akuter Myokarditis aussprachen. Prof. Friedrich betonte allerdings, dass die Lake-Louise Kriterien auch nur für die akute Myokarditis validiert seien. PD Dr. Dr. Philip Lurz (Leipzig) vertrat die Position, dass zwar die Biopsie ihre deutlichen Limitationen habe, aber insbesondere bei Studien von Patienten mit dem Verdacht auf eine chronische Myokarditis, wo die klinischen Kriterien nicht mehr eindeutig seien, eine Biopsie als Referenz zu fordern sei.

Das Mapping kann aber auch genutzt werden, um verschiedene hypertrophe Kardiomyopathien voneinander zu unterscheiden. Insbesondere Patienten mit einem M. Fabry scheinen niedrigere T1-Werte zu haben als Patienten mit einer hypertrophen Kardiomyopathie oder mit anderen infiltrativen Erkrankungen [3, 4]. Das Mapping bietet zudem große Möglichkeiten, auch frühe myokardiale Veränderungen, wie zum Beispiel bei HFpEF [5], Aortenklappenstenose oder anderen myokardialen Erkrankungen, früh vor klinischen manifesten Veränderungen detektieren zu können.

Die initiale Hoffnung, dass die parametrische Bildgebung ähnlich robust wie die Houndsfield-Einheiten in der CT einsetzbar seien, scheint sich allerdings wohl leider nicht zu bestätigen.

Ein weiteres Highlight des Meetings stellten wieder die MRT- und CT-FACE-OFF-Sessions dar, bei der verschiedene Analyse-Softwarefirmen 2 Fälle innerhalb eines limitierten Zeitfensters von nur 7-10 Minuten auszuwerten hatten. Diesmal war neben der Auswertung einer MR-Stressuntersuchung eines Patienten mit KHK, die Shuntquantifizierung eines ASD gefragt ( $\triangleright$ Abb. 4). In der MR-Session traten 7 Firmen, in der CT-Session 5 Fir-

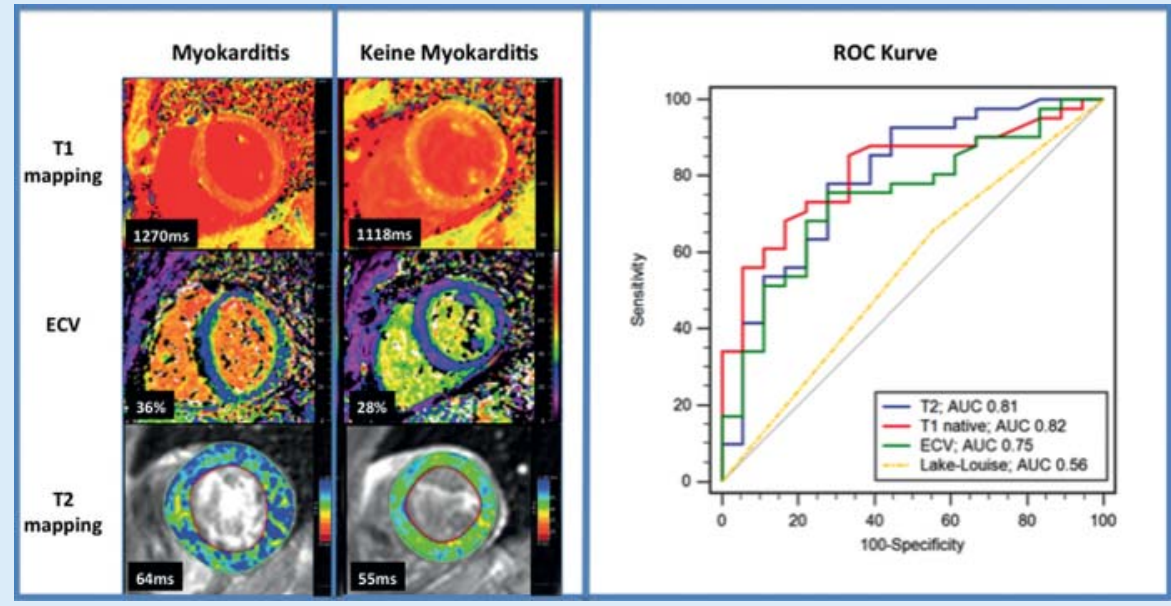

- Abb. 3 Links: T1 Zeiten auf 3.0 Tesla, berechnetes extrazelluläres Volumen sowie T2 Zeiten eines Patienten ohne (linke Spalte) und einen Patienten mit bioptisch gesicherter Myokarditis (rechte Spalte) mit höheren Werten bei Myokarditis für alle 3 Mapping-Parameter. Rechts: Receiver operator Kurven (ROC) von 61 Patienten mit V.a akute Myokarditis mit höheren AUCs für das native $\mathrm{T} 1$ mapping, berechnetes extrazelluläres Volumen $(0,75)$ sowie $\mathrm{T} 2$ mapping verglichen mit den Lake-Louise-Kriterien (adaptiert von Referenz 2).
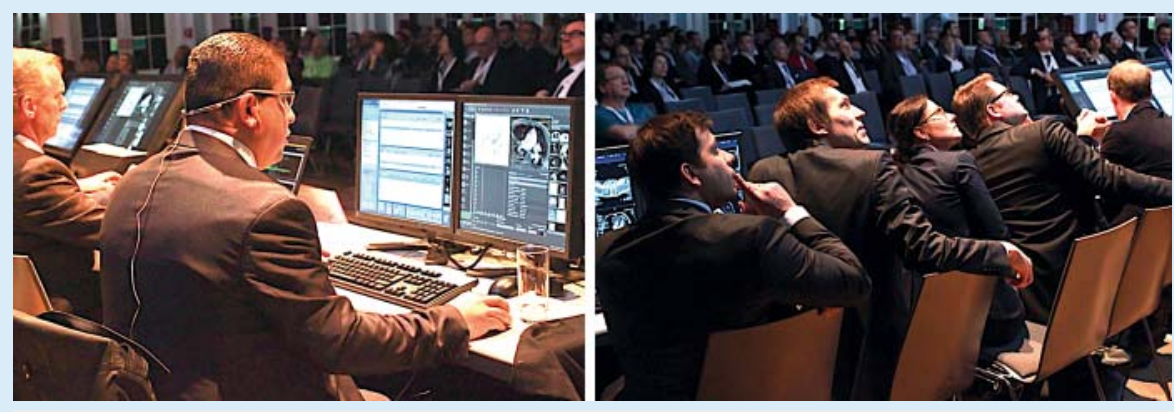

- Abb. 4 Links: Zwei Firmenteilnehmer an der MR FACE-OFF Session bei der Flussauswertung eines Patienten mit ASD und einem ausgeprägten Links-Rechtsshunt. Rechts: Gespannte Betrachtung der Auswertungen der Mitbewerber bei der MR FACE-OFF Session auf den zwei großen Leinwänden.

men "gegeneinander“ an. Hier musste neben einer Koronar-CT eine komplette PräTAVI-Evaluation erfolgen.

Die wissenschaftliche Leitung des Kongresses hat sich entschlossen auch im nächsten Jahr die Veranstaltung wieder in Leipzig durchzuführen. Dieses wird eine Jubiläumsveranstaltung werden, da es dann die 10. Deutschen Kardiodiagnostiktage sind, die vom 22.02.24.02.2018 wieder in der Kongresshalle im Zentrum der Stadt Leipzig stattfinden. Hier werden sich die Veranstalter sicher einiges Besonderes einfallen lassen. Weitere Infos unter: http://www.kardiodiagnostik.de/

\section{Referenzen}

[1] Friedrich MG, Sechtem U, Schulz-Menger ] et al. Cardiovascular magnetic resonance in myocarditis: A JACC White Paper. J Am Coll Card 2009; 53 (17): 1475-1487

[2] Lurz P, Luecke C, Eitel I et al. Comprehensive cardiac magnetic resonance imaging in patients with suspected myocarditis - The MyoRacerTrial. J Am Coll Card 2016; 67: 1800 - 1811

[3] Sado D, White S, Piechnik $S$ et al. The identification and assessment of Anderson Fabry disease by cardiovascular magnetic resonance non-contrast myocardial $\mathrm{T} 1$ mapping. Circ Cardiovasc Imaging 2013; 6: 392-398

[4] Sado DM, Flett AS, Banypersad SM et al. Cardiovascular magnetic resonance measurement of myocardial extracellular volume in health and disease. Heart 2012; 98: 1436 - 1441

[5] Rommel KP, von Roeder M, Latuscynski K et al. Extracellular Volume Fraction for characterization of patients with Heart Failure and preserved ejection fraction. J Am Coll Cardiol 2016; 67: 1815-1825 